\title{
Retraction: Developing Write-Back Caches and Information Retrieval Systems with EASEL
}

\author{
Mingqian Wang, Yingying Wang, Yueou Ren and Xi Zhao
}

Several conference proceedings have been infiltrated by fake submissions generated by the SCIgen computer program. Due to the fictional content the chapter "Developing Write-Back Caches and Information Retrieval Systems with EASEL" by "Mingqian Wang, Yingying Wang, Yueou Ren and Xi Zhao" has been retracted by the publisher. Measures are being taken to avoid similar breaches in the future. 